\title{
Expansion of ruminant-specific microRNAs shapes target gene expression divergence between ruminant and non-ruminant species
}

Hua Bao, Arun Kommadath, Xu Sun, Yan Meng, Adriano S Arantes, Graham S Plastow, Le Luo Guan* and Paul Stothard

\begin{abstract}
Background: Understanding how species-specific microRNAs (miRNAs) contribute to species-specific phenotypes is a central topic in biology. This study aimed to elucidate the role of ruminant-specific miRNAs in shaping mRNA expression divergence between ruminant and non-ruminant species.

Results: We analyzed miRNA and mRNA transcriptomes generated by Illumina sequencing from whole blood samples of cattle and a closely related non-ruminant species, pig. We found evidence of expansion of cattle-specific miRNAs by analyzing miRNA conservation among 57 vertebrate species. The emergence of cattle-specific miRNAs was accompanied by accelerated sequence evolution at their target sites. Further, the target genes of cattle-specific miRNAs show markedly reduced expression compared to their pig and human orthologues. We found that target genes with conserved or non-conserved target sites of cattle-specific miRNAs exhibit reduced expression. One of the significantly enriched KEGG pathway terms for the target genes of the cattle-specific miRNAs is the insulin signalling pathway, raising the possibility that some of these miRNAs may modulate insulin resistance in ruminants.

Conclusions: We provide evidence of rapid miRNA-mediated regulatory evolution in the ruminant lineage. Cattlespecific miRNAs play an important role in shaping gene expression divergence between ruminant and nonruminant species, by influencing the expression of targets genes through both conserved and cattle-specific target sites.
\end{abstract}

\section{Background}

It has long been hypothesized that differences in gene expression contribute extensively to phenotypic differences among species [1,2]. Numerous studies have investigated the effects of cis-acting elements and trans-acting proteins on gene expression divergence [3-5]. A recently discovered class of regulatory RNA molecules called miRNAs is known to play an important role in gene expression. It is now predicted that nearly $50 \%$ of mammalian mRNAs are regulated at the translational level by miRNAs [6,7]. Many miRNAs exhibiting both broad sequence and expression conservation among animal species have been identified $[8,9]$. However, recent high-throughput small RNA sequencing and comparative genomic studies have led to the discovery of a large number of miRNAs with limited

\footnotetext{
*Correspondence: Iguan@ualberta.ca; stothard@ualberta.ca

Department of Agricultural, Food and Nutritional Science, University of Alberta, Edmonton, Alberta, Canada
}

(c) 2013 Bao et al.; licensee BioMed Central Ltd. This is an Open Access article distributed under the terms of the Creative Commons Attribution License (http://creativecommons.org/licenses/by/2.0), which permits unrestricted use, distribution, and reproduction in any medium, provided the original work is properly cited. by these miRNAs, some of which may be species-specific, may be one of the important mechanisms behind some of the expression and phenotype divergence observed among species.

In this study, we aimed to investigate how speciesspecific miRNAs drive gene expression divergence by identifying cattle-specific miRNAs and characterizing their contribution to cattle-specific gene expression divergence using Illumina sequencing and comparative genomics. Dramatic physiological and phenotypic differences exist between ruminant and non-ruminant mammalian species. For example, volatile fatty acids produced as by-products of the microbial fermentation in the rumen are used as the major source of energy in ruminants as opposed to glucose absorbed from the small intestine in non-ruminants. Because of this difference in nutrient usage, ruminants are less sensitive to insulin than 
non-ruminants [18]. Several major genes involved in the insulin pathway, including INSR, GLUT1, GLUT4 and PI3K, show decreased expression in ruminants compared to non-ruminants [19-21].

\section{Results}

Profiling of miRNAs from the whole blood of cattle and pigs To assess miRNA repertoire divergence between a ruminant and a closely related non-ruminant species, we sequenced miRNAs isolated from whole blood samples of cattle and pigs. From three blood samples of each species, an average of 26 million reads (per sample) (Additional file 1) were obtained, and approximately $89 \%$ of the reads could be mapped to the corresponding reference genome assemblies: UMD3.1 for cattle and Sscrofa10.2 for pigs. A total of 676 and 257 miRNAs have been reported for cattle and pigs respectively in miRBase release 18 . Using the miRNA discovery software, miRDeep2 [22], we identified at total of 228 and 148 known (previously reported in miRBase) miRNAs expressed in the blood of cattle and pig respectively (Table 1). The miRDeep2 software can also predict novel miRNAs using a probabilistic model of miRNA biogenesis to score compatibility of the position and frequency of sequenced RNA with the secondary structure of the miRNA precursor. The de novo prediction strategy employed by miRDeep 2 was able to identify $87 \%$ and $90 \%$ of the miRNAs observed in cattle and pig respectively through mapping to known miRNAs in miRBase. It was estimated that a miRDeep2 score cutoff of 5 corresponds to a true positive prediction percentage greater than $90 \%$, and a signal-to-noise ratio $>10$, criteria that the authors of the software recommend [22]. With these criteria, we predicted 33 and 54 novel miRNAs in cattle and pig respectively (Table 1 ). Thus a substantially higher number of miRNA species were identified in cattle blood compared to pig blood, and several of the

Table 1 Numbers of miRNAs identified from cattle and pigs

\begin{tabular}{lccc}
\hline Sample ID & Known & Novel & Total \\
\hline Cattle & 245 & 48 & \\
bta-1064 & 258 & 53 & 293 \\
bta-1146 & 250 & 51 & 311 \\
bta-1151 & 228 & 33 & 261 \\
All miRNAs & & & \\
Pig & 169 & 94 & 263 \\
ssc-404 F & 166 & 112 & 278 \\
ssc-406 F & 153 & 70 & 223 \\
ssc-422 F & 148 & 54 & 202 \\
All miRNAs & & &
\end{tabular}

${ }^{*}$ Refers to miRNAs with greater than 5 reads in all 3 samples.
miRNAs identified in this work have not been previously described. The number of reads detected for each of the known miRNAs is summarized in Additional file 2 and the sequences and genomic positions of novel miRNAs are shown in Additional file 3.

\section{Expansion and diversification of cattle-specific miRNAs}

Since a substantially higher number of expressed miRNAs were identified in cattle relative to pigs, we wondered if cattle possess additional miRNA genes not found in nonruminant genomes. To investigate this possibility, we analyzed the conservation of cattle miRNAs across 57 vertebrate species with good quality genome assemblies available in the Ensembl database, version 71 (http://www.ensembl.org/index.html). Our analyses revealed an overall expansion of miRNA repertoire in cattle. About 20\% (46 of 228) of the known and over $76 \%$ ( 25 of 33) of the novel cattle miRNAs did not have homologs in any of the 57 vertebrate species (Table 2). We refer to these 71 miRNAs as 'cattle-specific' (read counts provided in Additional file 4). The miR-2284 family with 24 members reported in miRBase 18 is the largest miRNA family in cattle. Based on our conservation analysis, this family is also cattle-specific.

After a new miRNA gene has emerged, through duplication of an existing gene for example, it can be further diversified in different ways. A seed region of about 7 nucleotides in length at the $5^{\prime}$ end of an animal miRNA is thought to be an important determinant of target specificity [23]. One of the mechanisms for acquiring divergent function is seed shifting, wherein the dominant mature miRNA isoform is shifted by one to several nucleotides in the $5^{\prime}$ or $3^{\prime}$ direction relative to its original position $[8,14,24]$. The miR-2284 family with 24 members provides us with an opportunity to investigate how different mechanisms shaped its diversification. An alignment of the sequences of the members of this family reveals that they share only 13 seed sequences and that these seeds might have evolved by seed shifting and point mutation (Figure 1).

Based on the numbers of reads obtained for each miRNA, most of the 71 cattle-specific miRNAs are expressed at a low level relative to the non-cattle-specific miRNAs, at least in blood (Figure 2). Of these 71, we consider 33 miRNAs with read count greater than 50 (corresponding to $\mathrm{cpm}$ above 1.5) as relatively highly expressed cattle-specific miRNAs. These 33 miRNAs

Table 2 Conservation of cattle miRNAs across vertebrate species

\begin{tabular}{lccc}
\hline Category & Known & Novel & All \\
\hline Cattle-specific miRNAs & 46 & 25 & 71 \\
Non-cattle-specific miRNAs & 182 & 8 & 190 \\
\hline
\end{tabular}




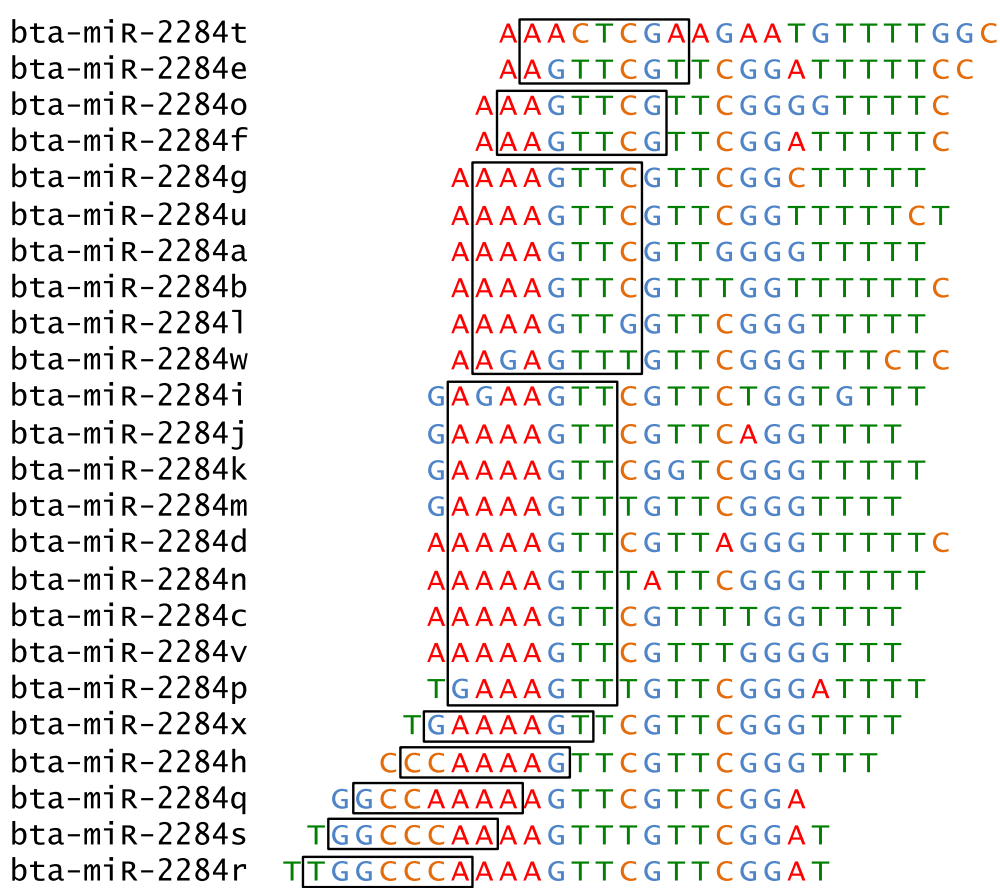

A AACTCGAAGAATGTTTTGGC A A GTTCGTTCGGATTTTTCC A AAGTTCGT T C G GGGTTTTC A AAGTTCGTTCGGATTTTTC A AAAGTCG TTCGGCTTTTT A AAAGTTCGTTCGGTTTTTCT A A AAGTTCGTTGGGGTTTTT A AAAGTTCGTTTGGTTTTTTC AAAAGTTGGTTCGGGTTTTT A A GAGTTTGTTCGGGTTTCTC GAGAAGTTCGTTCTGGTGTTT GAAAAGTTCGTTCAGGTTTT GAAAAGTTCGGTCGGGTTTTT GAAAAGTTTGTTCGGGTTTT A A AAAGTTCGTTAGGGTTTTTC A A A A A GTTTATTCGGGTTTTT AAAAAGTTCGTTTTGGTTTT A A A A A GTTCGTTTGGGGTTT TGAAAGTTTGTTCGGGATTTT

TAAAAGTTCGTTCGGGTTTT

C CAAAAGT TCG T T C G G GTT T

GCCAAAAAGTTCGTTCGGA

TGGCCCAAAAGTTTGTTCGGAT TTGGCCCAAAAGTTCGTTCGGAT

Figure 1 Evolution of the miR-2284 family by seed shifting and point mutation. Alignment of the mature cattle miRNA sequences in the mir-2284 family shows the presence of sequence substitutions and differences in the position of the seed region (positions 2-8, boxed). mir-2284 family shows the presence of sequence substitutions and differences in the position of the seed region (positions $2-8$, boxed).

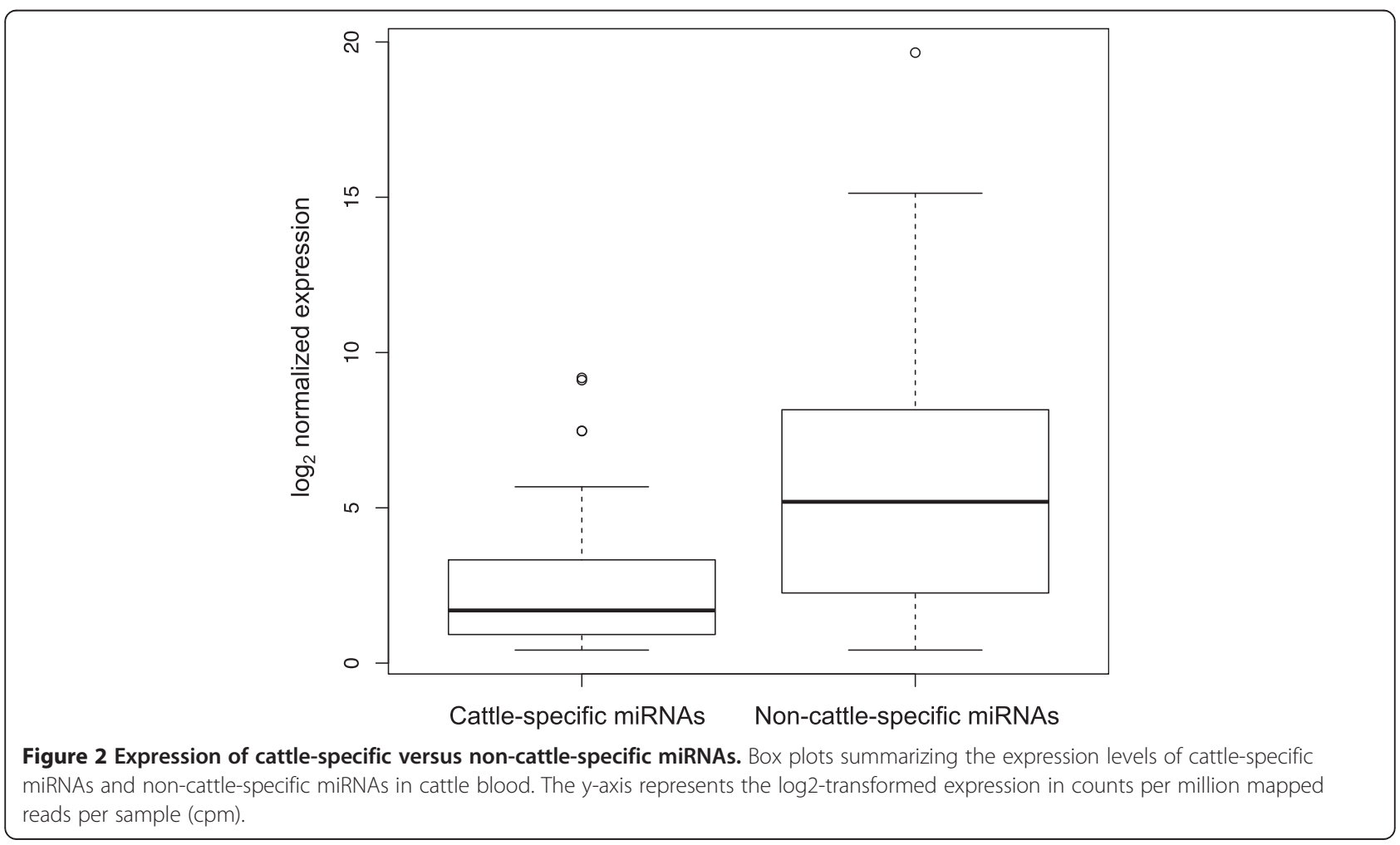


included 14 members of the miR-2284 family of which bta-miR-2284x had the highest expression.

\section{Target sites of cattle-specific miRNAs show accelerated sequence evolution}

To better understand how target sites have evolved with the emergence of cattle-specific miRNAs, we first evaluated whether the cattle-specific miRNAs have a greater proportion of targets that are also cattle-specific. We used the predicted target sites of known and novel miRNAs from TargetScan (see Methods) and determined their positions on the 23-way UTR alignments available in TargetScan. Indeed a significantly higher $(\mathrm{p}<0.0001$, chi-square test) proportion of target sites were found to be specific to cattle $(60 \%)$ for cattle-specific miRNAs compared to that (49\%) for non-cattle-specific miRNAs (Table 3). Next, we compared the distribution of normalized divergence rates (defined as the divergence rate of the target site minus the divergence rate of the flanking regions) of target sites for cattle-specific versus non-cattle-specific miRNAs. The distribution for cattlespecific miRNAs displayed a shift towards the right indicating a significantly higher divergence rate $(\mathrm{p}<2.2 \mathrm{e}-16$, Kolmogorov-Smirnov test) and thus more rapid sequence evolution for target sites of cattle-specific miRNAs relative to those of non-cattle-specific miRNAs (Figure 3). The same trends were obtained when the context + score cutoff for predicting miRNA targets was relaxed (context + score cutoff $=90$ ) or made more stringent (context + score cutoff $=99$ ) indicating that the results were not dependent on the choice of context + score cutoffs. Thus we demonstrate that the emergence of cattle-specific miRNAs was accompanied by accelerated sequence evolution of their target sites.

\section{Decreased expression of the target genes of cattle- specific miRNAs}

Although our analyses suggest that there have been substantial additions to the miRNA and miRNA target repertoire during the evolution of cattle relative to pigs and other mammals, an important question remaining in this study is whether cattle-specific miRNAs contribute to gene expression divergence. To test this we measured mRNA expression in the whole blood of seven cattle and

\section{Table 3 Targets of cattle-specific and non-cattle-specific} miRNAs

\begin{tabular}{cccc}
\hline miRNA type & \multicolumn{3}{c}{ Predicted target type } \\
\cline { 2 - 4 } & Cattle-specific & Conserved & Total \\
\hline Cattle-specific & $6937(60 \%)$ & 4535 & 11472 \\
Non-cattle-specific & $13425(49 \%)$ & 13969 & 27394 \\
\hline
\end{tabular}

The predicted targets information is available from TargetScan for only the major miRNA sequences (not for the minor (star) sequences) i.e. 65 of the 71 cattle-specific and 183 of the 190 non-cattle-specific miRNAs. three pigs using RNA-seq. We used the distribution of expression ratios of all orthologous genes between cattle and pig as the genome-wide background $(\mathrm{n}=8680)$. The target genes of 33 highly expressed cattle-specific miRNAs were indeed expressed at lower levels in cattle relative to their pig orthologues, as indicated by a leftward shift in the cumulative distribution of the mRNA expression ratio for the highly expressed cattle-specific miRNAs relative to the genome-wide background ( $\mathrm{p}=1.014 \mathrm{e}-15$, KolmogorovSmirnov test) (Figure 4A). Next we tested whether cattlespecific miRNAs tend to decrease target gene expression by binding to cattle-specific or conserved target sites. Targets of cattle-specific miRNAs with conserved target sites and those with cattle-specific target sites showed reduced expression in cattle relative to the genome-wide background ( $\mathrm{p}=1.118 \mathrm{e}-11$ and $1.124 \mathrm{e}-10$ respectively, Kolmogorov-Smirnov test) (Figure 4B). The distributions for the target genes of cattle-specific miRNAs with conserved target sites (blue line in Figure 4B) were not significantly different $(\mathrm{p}=0.4$, Kolmogorov-Smirnov test) from those with cattle-specific target sites (red line in Figure 4B). We observe a similar pattern of expression divergence when using an expression data set from human blood (GEO Series accession number GSE33701) [25] as the one we generated from pigs (Additional file 5).

We next looked at the magnitude of expression reduction of targets of highly expressed miRNAs compared to genome-wide background. We only looked at the genes with fold change between pig and cattle greater than 1.2. The targets of the highly expressed cattle-specific miRNAs (median $\operatorname{logFC}=-1.73$ ) showed significantly more reduction ( $\mathrm{p}=0.0065$, Mann-Whitney $\mathrm{U}$ test) than the genome-wide background (median $\operatorname{logFC}=-1.60$ ) (Figure 4C). Thus the target genes of highly expressed cattle-specific miRNAs showed 8\% ((1.73-1.60)/1.60) greater expression reduction compared to the genomewide background. Dividing the targets of the highly expressed cattle-specific miRNAs into those having cattle-specific or conserved target sites, we found that the degree of reduction in expression for both types (median $\log \mathrm{FC}=-1.71$ and -1.77 respectively) was significantly greater $(\mathrm{p}=0.052$ and 0.012 respectively, Mann-Whitney $U$ test) than the genome-wide background (Figure 4D). The reduction in expression of target genes with conserved target sites of the highly expressed cattle-specific miRNAs was not significantly different from the target genes with cattle-specific targets ( $p>0.05$, Mann-Whitney U test).

\section{Functional enrichment of target genes of cattle-specific miRNAs}

In order to assess the biological effects of the 33 highly expressed cattle-specific miRNAs, we looked for enriched biological pathways among the genes they target. We 


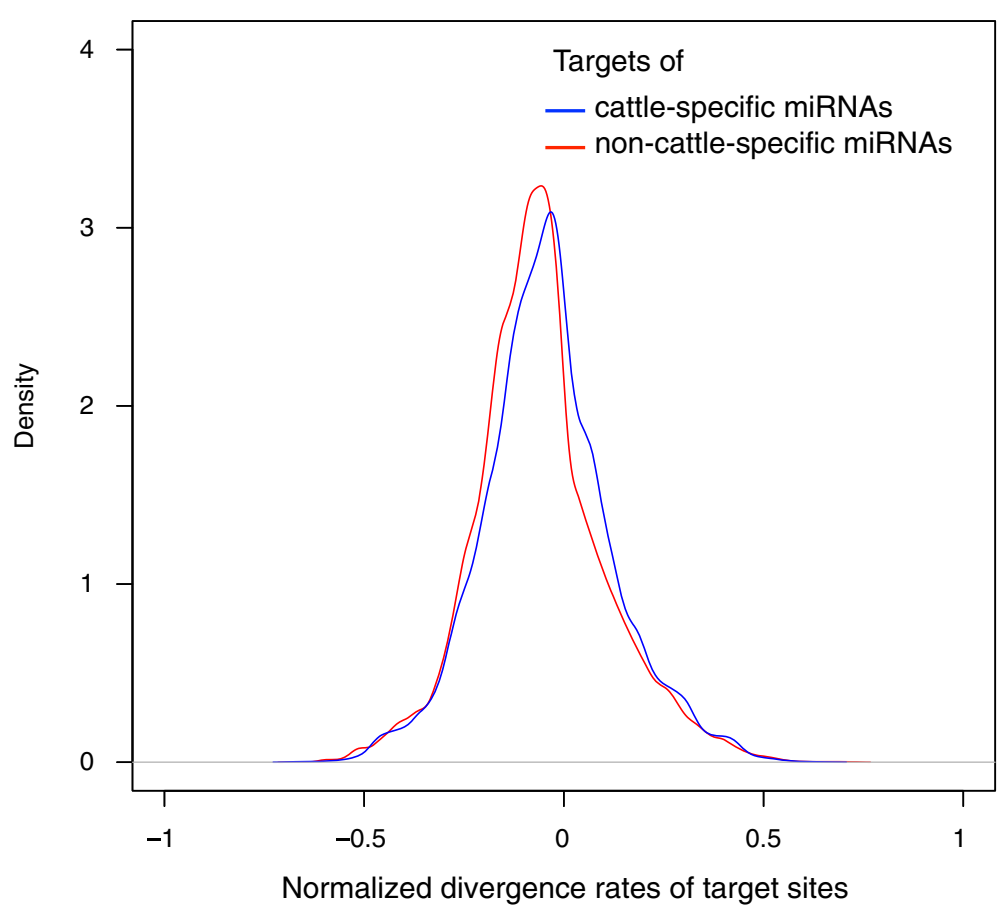

Figure 3 The distribution of normalized divergence rates of miRNA target sites. Density plots of the normalized target site divergence rates between cattle and human for cattle-specific (blue line) and non-cattle-specific miRNAs (red line). The targets of the cattle-specific miRNAs show a greater normalized divergence rate than the targets of the non-cattle-specific miRNAs ( $p=2.2 e-16$, Kolmogorov-Smirnov test).

examined KEGG pathway enrichment for targets expressed in blood $(\mathrm{n}=1708)$ and for expressed targets with reduced expression in cattle compared to pigs $(\mathrm{n}=856)$ and human $(\mathrm{n}=754)$. Because miRNAs expressed in blood can target genes in other tissues [26], we also looked at all predicted targets $(n=3255)$ irrespective of their expression in blood. Although most of the KEGG pathways found to be enriched $(p<0.05$ and gene count $>=3)$ did not show an obvious relationship to cattle-specific functions (Additional file 6), the insulin signalling pathway, which is known to contribute to metabolic differences between ruminants and non-ruminants, is enriched in targets expressed in blood $(\mathrm{p}=0.044)$ and in expressed targets with reduced expression in cattle compared to human $(\mathrm{p}=0.032)$ but not pig $(\mathrm{p}=0.165)$. The insulin signalling pathway showed a p-value of 0.059 when considering all predicted targets of the 33 highly expressed cattle-specific miRNAs. Notably, cattle-specific miRNAs may target some of the key annotated insulin signalling pathway genes, including AKT3, CBLB, FOXO1 and PIK3R5 (all show reduced expression in cattle compared to human and pig).

\section{Discussion}

In this study, $23 \%$ of the miRNAs identified from cattle whole blood are found to have no homologs in 57 other vertebrate species examined. Based on this set of cattlespecific miRNAs, we can provide an estimate of the net gain rate of new miRNAs during cattle evolution. Given the estimated 64.5 Myr (million years) divergence time between cattle and pig [27] and the 71 cattle-specific miRNAs we identified, the net gain rate of miRNAs expressed in blood is estimated as 1.1 miRNAs per Myr. This is about twice the rate of that observed in pigs ( 0.6 miRNAs per Myr). One of the most interesting cases is the bta-mir-2284 family, which has 24 members. Why does the cattle genome maintain so many members in this family? The abundant miRNA seeds generated by seed shifting and point mutation in this family indicate that the emergence of novel miRNAs may have led to adaptive functional diversification. However, the number of unique seeds is much less than the number of paralogues and many miRNA members share the same seed sequence, suggesting that dosage effect might be also important for the function of mir-2284 family.

It has long been hypothesized that gene expression changes are one of the main underlying causes of phenotypic differences between species $[1,2]$. While divergence in cis-acting elements and trans-acting proteins has been shown to underlie evolutionary divergence [3,4], relatively little is known about the role of miRNAs in shaping gene expression divergence. Here we showed that both the proportion of genes with decreased expression and the degree of expression reduction (relative to their pig and human orthologues) are higher for targets of cattle-specific miRNAs compared to genome-wide background. The 


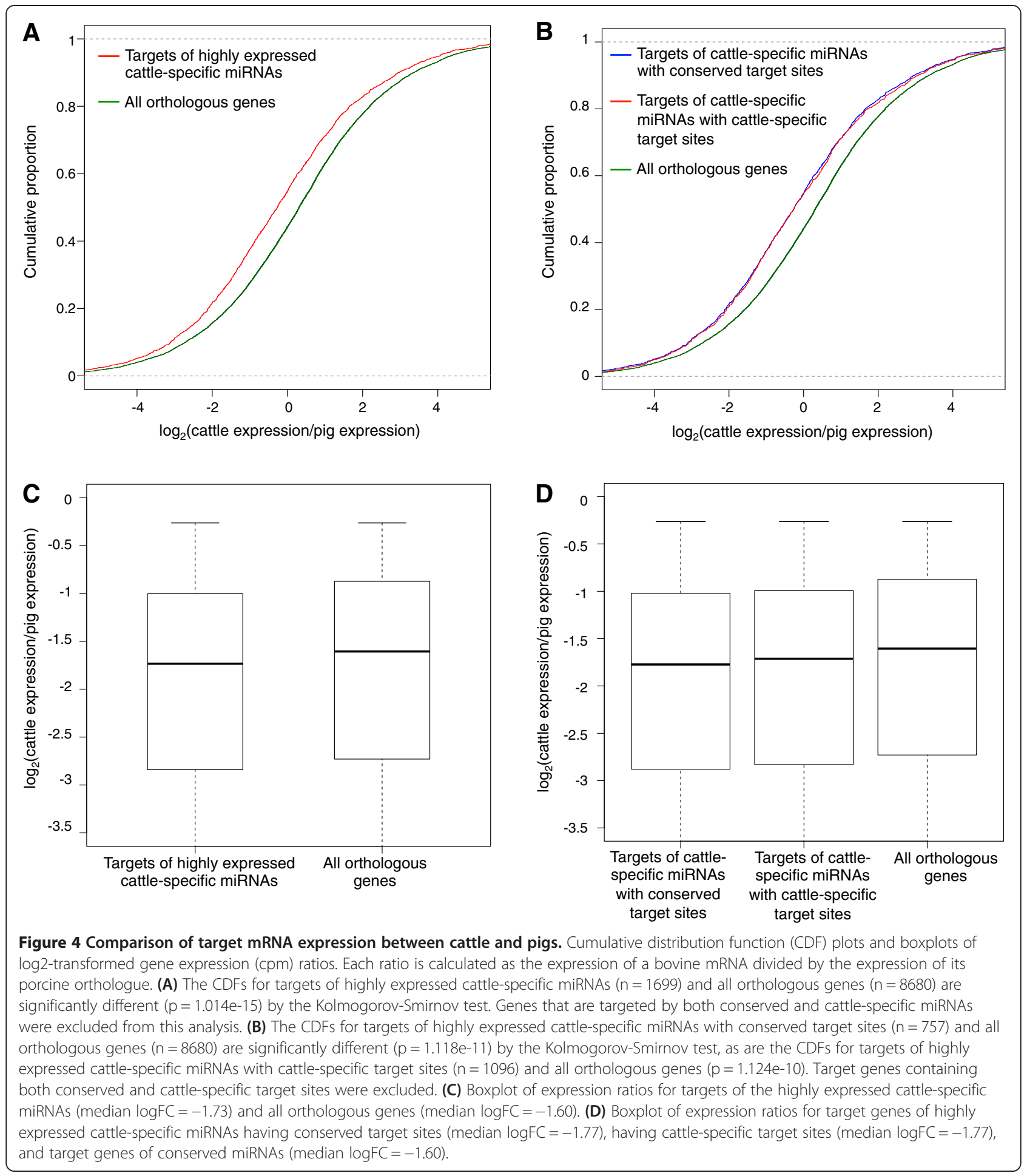

target genes of cattle-specific miRNAs might have been under selection for decreased expression, which has been achieved by several means, one of them being cattlespecific miRNAs. However, the fact that the target genes of highly expressed cattle-specific miRNAs show a greater reduction in expression than those of the cattle-specific
miRNAs expressed at low levels (Additional file 7) further implicates miRNAs as the major player in shaping the expression patterns of these genes, as opposed to other factors. Functionally, cattle-specific miRNAs might be associated with the insulin signalling pathway, and thus potentially have a role in the evolution of insulin resistance 
in ruminants. It would be worthwhile to analyse how species-specific miRNAs modulate target gene expression divergence across other model animal species for speciesspecific functions.

In this study, we found more target genes with cattlespecific target sites for cattle-specific miRNAs than for non-cattle-specific miRNAs and we observed accelerated sequence evolution of target sites of cattle-specific miRNAs. This accelerated evolution suggests that selection might have favoured the formation of new target sites. Previous studies have primarily focused on conserved target sites but our findings suggest that the nonconserved targets may represent novel mechanisms of genetic regulation that can contribute to species-specific phenotype. Based on target gene expression analyses, we showed that cattle-specific miRNAs have effects on targets genes of both types: those with conserved targets sites and those with cattle-specific target sites. Thus these miRNAs may fine tune pre-existing regulatory mechanisms as well as contribute to the formation of new regulatory mechanisms.

\section{Conclusions}

We provide evidence of rapid miRNA-mediated regulatory evolution in the ruminant lineage. Cattle-specific miRNAs play an important role in shaping gene expression divergence between ruminant and non-ruminant species, by influencing the expression of target genes with either conserved or cattle-specific target sites. One interesting potential role for these miRNAs is to increase insulin resistance in ruminants by targeting insulin signalling.

\section{Methods}

\section{Sample collection and RNA preparation}

Three cattle and three pig blood samples were used for miRNA sequencing. One pooled sample from seven cattle blood samples and three separate pig blood samples were used for mRNA sequencing. Peripheral whole blood (approximately $2.5 \mathrm{~mL}$ ) was collected from the jugular vein of cattle and pigs, into PAXgene Blood RNA tubes (BD, Cat. No. 762165) and processed according to the manufacturer's instructions. Total RNA was extracted from $4.5-9.0 \mathrm{ml}$ of solution from the PAXgene Blood RNA tubes using the PreAnalytiX kit (Qiagen, Cat. No. 763134). The quality and quantity of the RNA were determined using Agilent 2100 Bioanalyzer (Agilent Technologies, Santa Clara, CA) and Qubit 2.0 Fluorometer (Invitrogen, Carlsbad, CA). The animal study was approved by the Animal Care and Use Committee of the University of Alberta (Guan 019).

\section{Library construction and Illumina sequencing}

Total RNA (1.5 $\mu \mathrm{g}$ for each sample) was used to construct miRNA and mRNA libraries using the TruSeq Small RNA and mRNA Sample Preparation Kit (Illumina, San Diego, CA) according to the manufacturer's instructions. PCR amplification was performed for 12 cycles. Library quality for miRNA and mRNA libraries was determined using the High Sensitivity DNA Chip and an Agilent 2100 Bioanalyzer (Agilent Technologies). qRT-PCR was then performed for library quantification using the StepOne ${ }^{\text {TM }}$ Real-Time PCR System (Applied Biosystems, Carlsbad, CA) with the KAPA SYBR * Fast ABI Prism qPCR kit (Kapa Biosystems, Woburn, MA).

The individual libraries were adjusted to $2 \mathrm{nM}$ concentrations and pooled before denaturation and dilution according to Illumina's instructions. The diluted libraries $(8-10 \mathrm{pM})$ were loaded on a cBot (Illumina) for cluster generation using the TruSeq ${ }^{\text {тм }}$ SR Cluster Kit v3 (Illumina). Sequencing was performed on the HiScan SQ system (Illumina) using the TruSeq ${ }^{\mathrm{Tm}}$ SBS Kit v3 (50 cycels, Illumina). Real-time analysis and base calling was performed using the HiSeq Control Software version 1.4.8 (Illumina).

\section{Identification and quantification of known and novel miRNAs}

Sequence reads with base quality scores were produced by the Illumina sequencer. Reads flagged as low-quality by CASAVA 1.8 were removed. After trimming the 3' adaptor sequence, all sequences ranging in length from 18-26 nt were recorded in a non-redundant file along with copy number. Further analyses were performed using miRDeep2 [22,28] and custom Perl scripts. All tags were compared to the Rfam database of RNA families $[29,30]$ to filter out non-miRNA sequences. To identify known miRNAs, the miRNA tags of the six samples were aligned against miRNA precursor sequences reported in the miRNA database 'miRBase' (release 18) [31-34] using the 'quantifier.pl' script (with the default parameters) within miRDeep2. Candidates for novel miRNAs were identified by miRNA precursor prediction within miRDeep2. For novel miRNA prediction, the following criteria as recommended by the authors of miRDeep2 [22] were chosen: miRDeep score cutoff of 5, which is estimated by miRDeep 2 to yield a true positive rate $>90 \%$ and a signal-to-noise ratio $>10$. Each sample was processed separately and the results for each species were combined by genomic location. We considered only those miRNAs with read counts greater than five in all three samples as being expressed. The read counts per miRNA in each sample were normalized to counts per million mapped reads (cpm).

\section{Identification of miRNA homologs}

A three-step procedure was used to find homologs of the known and novel cattle miRNAs identified. First, the mature sequences of the cattle miRNAs were aligned 
against the genomes of 57 vertebrate species with good quality assembled genomes available in the Ensembl database version 71 (http://www.ensembl.org/index.html) [35] using the short read aligner software Bowtie [36]. The hairpin (precursor) sequences of the cattle miRNAs with mature sequences that showed no more than two mismatches in the previous alignment step were then selected for a second round of alignments against the 57 vertebrate genomes. The nucleotide blast program (blastn) of NCBI's 'Basic Local Alignment Search Tool' (BLAST) [37] was used to perform this alignment, with cutoffs for expected score set at below 0.1 and percent identity set at above $60 \%$ of the length of the hairpin sequence. Finally, the stabilities of the secondary structures of the predicted homologous hairpin sequences were tested based on their minimum free energy of folding (below $-25 \mathrm{kcal} / \mathrm{mol}$ ) using the RNA secondary structure prediction program RNAfold [38] within the Vienna RNA package (version 2.0) [39]. The cattle miRNAs with no homolog in any other species were classified as "cattle-specific" and those with a homolog in any other species were classified as "non-cattle-specific".

\section{Sequence conservation of target seed sites}

To evaluate the conservation of target seed sites of miRNAs across species, we used the predicted miRNA target sites with context + scores above $95^{\text {th }}$ percentile and determined their positions on the 23-way UTR alignments available in TargetScan (release 6.2, June 2012) [40-42]. The target predictions in TargetScan are made only for the major sequence and not the minor (star) sequence of the miRNAs. We used the Perl scripts provided on the TargetScan website (http://targetscan.org) for predicting and calculating the context scores for the targets of novel miRNAs.

For each cattle miRNA target seed site the aligned sequences from five other species available in the 23-way UTR alignments were examined: human, dog, mouse, rat and chicken (the pig genome was not available in this multi-species alignment). If the target site was not perfectly conserved with any of the five species considered (both nucleotide substitutions and indels in the other species were considered as divergence), then the target site was classified as cattle-specific.

We used the method described by Zheng et al. [43] to calculate the normalized divergence rates of target sites between cattle and human. The normalized divergence rate is defined as the divergence rate of the target site minus the divergence rate of flanking region. The divergence rate of the target site is defined as the number of nucleotide substitutions in the target sequence divided by its length (7-mer or 8-mer) and the divergence rate of the flanking regions is defined as the number of nucleotide substitutions in the upstream and downstream regions, divided by the corresponding flanking region lengths (84 and 96 respectively for flanking regions of 7-mer and 8-mer seed sequences).

\section{Comparison of expression levels of cattle mRNAs with their orthologues in pig and human}

Sequence reads with base quality scores were produced by the Illumina sequencer. RNA-seq reads flagged as low-quality by CASAVA 1.8 were removed. Cattle and pig reads were aligned to the cattle (UMD 3.1) and pig (Sus 10.2) reference genome sequences respectively using Tophat 1.4 .0 with default parameters [44]. The number of reads mapped to each gene was determined using htseq-count (v0.5.3.p3). The read counts per gene were normalized to $\mathrm{cpm}$.

The pig and human orthologues of cattle mRNAs were determined using the homology mappings provided in the BioMart biological database (version 0.7) [45] (http://ensembl.org/biomart/martview/). A total of 8680 orthologous pairs between cattle and pig showed cpm $>0.5$ in both species and were used for downstream analyses. Similarly, we identified 9442 orthologous pairs between cattle and human. The RNA-seq-determined expression levels (expressed in cpm) of the cattle mRNA targets were then compared to those of their porcine and human orthologues for various subsets of targets, using cumulative proportion plots and Kolmogorov-Smirnov and MannWhitney U statistical tests.

\section{Test for biological pathway enrichment among target genes of highly expressed cattle-specific miRNAs}

The target genes (predicted by TargetScan with context + scores above $95^{\text {th }}$ percentile) of 33 highly expressed cattlespecific miRNAs were subjected to KEGG pathway [46] enrichment analysis using the 'GOstats' tool [47] within the Bioconductor package (version 2.12) of the R statistical programming language (version 3.0.1) [48]. Of the 390 KEGG terms from the 'KEGG.db' annotation map [49], we used 375 for the enrichment testing after excluding 15 cancerrelated terms. Conversions between bovine Ensembl IDs, Entrez gene IDs and gene symbols, when needed, were done using the genome wide annotation map for bovine available from the Bioconductor package 'org.Bt.eg.db' [50].

\section{Availability of supporting data}

The miRNA and mRNA sequence data sets described in this article are available from the NCBI Sequence Read Archive under accession ID SRP018740 at http://trace. ncbi.nlm.nih.gov/Traces/sra/?study=SRP018740.

\section{Additional files}

Additional file 1: Mapping statistics of miRNAs data. Spreadsheet contains the numbers of reads sequenced and mapped from cattle and pig miRNA libraries. 
Additional file 2: Known cattle and pig miRNAs identified by miRDeep2. Spreadsheet contains the numbers of reads mapped to known miRNAs.

Additional file 3: Novel cattle and pig miRNAs predicted by miRDeep2. Spreadsheet contains the position and sequence of novel miRNAs predicted by miRDeep2.

Additional file 4: Cattle-specific miRNAs identified in this work. Spreadsheet contains the known and novel cattle-specific miRNAs based on conservation analysis.

Additional file 5: Comparison of target mRNA expression between cattle and human. PDF file contains the cumulative distribution function (CDF) plots of log2-transformed gene expression (cpm) ratios.

Additional file 6: Enriched pathway for targets of cattle-specific miRNAs. Spreadsheet contains the KEGG pathway enriched among predicted targets of the highly expressed cattle-specific miRNAs.

Additional file 7: Comparison of mRNA expression for targets of highly versus lowly expressed cattle-specific miRNAs. PDF file contains the cumulative distribution function (CDF) plots of log2transformed gene expression (cpm) ratios.

\section{Competing interests}

The authors declare that they have no competing interests.

\section{Authors' contributions}

$\mathrm{HB}$ and $\mathrm{AK}$ conceived study, carried out the analyses and wrote the manuscript. XS and YM performed the experiment. ASA contributed to sequencing data management. GSP involved in discussion and manuscript revision. PS and LLG conceived and supervised the study, and revised the manuscript. All authors revised the manuscript and approved the final version.

\section{Authors' information}

Co-first authors; Hua Bao and Arun Kommadath.

\section{Acknowledgments}

This work is supported by the Applied Livestock Genomics Program (ALGP13) funded by Genome Alberta and Alberta Livestock and Meat Agency, and the Large-Scale Applied Research Project, CanadaCow, funded by Genome Canada. Graham Plastow, Leluo Guan, and Paul Stothard are grateful for the financial support of the Alberta Livestock and Meat Agency. The funders had no role in study design, data collection and analysis, decision to publish, or preparation of the manuscript

Received: 19 March 2013 Accepted: 6 September 2013

Published: 10 September 2013

\section{References}

1. Hu HY, Guo S, Xi J, Yan Z, Fu N, Zhang X, Menzel C, Liang H, Yang H, Zhao $M$, et al: MicroRNA expression and regulation in human, chimpanzee, and macaque brains. PLoS Genet 2011, 7:e1002327.

2. Somel M, Liu X, Tang L, Yan Z, Hu H, Guo S, Jiang X, Zhang X, Xu G, Xie G, et al: MicroRNA-driven developmental remodeling in the brain distinguishes humans from other primates. PLOS Biol 2011, 9:e1001214.

3. He F, Zhang X, Hu J, Turck F, Dong X, Goebel U, Borevitz J, de Meaux J: Genome-wide analysis of Cis-regulatory divergence between species in the Arabidopsis genus. Mol Biol Evol 2012, 29:3385-3395

4. Li C-M, Tzeng J-N, Sung H-M: Effects of cis and trans regulatory variations on the expression divergence of heat shock response genes between yeast strains. Gene 2012, 506:93-97.

5. Wunderlich Z, Bragdon MD, Eckenrode KB, Lydiard-Martin T, PearlWaserman S, DePace AH: Dissecting sources of quantitative gene expression pattern divergence between Drosophila species. Mol Syst Biol 2012, 8:604.

6. Flynt AS, Lai EC: Biological principles of microRNA-mediated regulation: shared themes amid diversity. Nat Rev Genet 2008, 9:831-842.

7. He L, Hannon GJ: MicroRNAs: small RNAs with a big role in gene regulation. Nat Rev Genet 2004, 5:522-531.
8. Wheeler BM, Heimberg AM, Moy VN, Sperling EA, Holstein TW, Heber S, Peterson KJ: The deep evolution of metazoan microRNAs. Evol Dev 2009, 11:50-68.

9. Saetrom $\mathrm{P}$, Snove $\mathrm{O}$, Nedland M, Grunfeld TB, Lin Y, Bass MB, Canon JR: Conserved microRNA characteristics in mammals. Oligonucleotides 2006, 16:115-144.

10. de Wit E, Linsen SE, Cuppen E, Berezikov E: Repertoire and evolution of miRNA genes in four divergent nematode species. Genome Res 2009, 19:2064-2074

11. Cuperus JT, Fahlgren N, Carrington JC: Evolution and functional diversification of MIRNA genes. Plant Cell 2011, 23:431-442.

12. Lu J, Shen Y, Wu Q, Kumar S, He B, Shi S, Carthew RW, Wang SM, Wu Cl: The birth and death of microRNA genes in Drosophila. Nat Genet 2008, 40:351-355.

13. Zhang R, Wang YQ, Su B: Molecular evolution of a primate-specific microRNA family. Mol Biol Evol 2008, 25:1493-1502.

14. Berezikov E: Evolution of microRNA diversity and regulation in animals. Nat Rev Genet 2011, 12:846-860.

15. Hu HY, He L, Fominykh K, Yan Z, Guo S, Zhang X, Taylor MS, Tang L, Li J, Liu J, et al: Evolution of the human-specific microRNA miR-941. Nat Commun 2012, 3:1145.

16. Berezikov E, Thuemmler F, van Laake LW, Kondova I, Bontrop R, Cuppen E, Plasterk RH: Diversity of microRNAs in human and chimpanzee brain. Nat Genet 2006, 38:1375-1377.

17. Zhang JF, He ML, Fu WM, Wang H, Chen LZ, Zhu X, Chen Y, Xie D, Lai $P$, Chen $G$, et al: Primate-specific microRNA-637 inhibits tumorigenesis in hepatocellular carcinoma by disrupting signal transducer and activator of transcription 3 signaling. Hepatology 2011, 54:2137-2148.

18. Brockman RP, Laarveld B: Hormonal regulation of metabolism in ruminants; a review. Livest Prod Sci 1986, 14:313-334.

19. Sasaki SI: Mechanism of insulin action on glucose metabolism in ruminants. Anim Sci J 2002, 73:423-433.

20. Sinclair KD: Declining fertility, insulin resistance and fatty acid metabolism in dairy cows: developmental consequences for the oocyte and preimplantation embryo. Acta Scientiae Veterinariae 2010, 38(Supl 2):\$545-\$557.

21. Saltiel AR, Kahn CR: Insulin signalling and the regulation of glucose and lipid metabolism. Nature 2001, 414:799-806.

22. Friedländer MR, Mackowiak SD, Li N, Chen W, Rajewsky N: miRDeep2 accurately identifies known and hundreds of novel microRNA genes in seven animal clades. Nucleic Acids Res 2012, 40:37-52

23. Brennecke J, Stark A, Russell RB, Cohen SM: Principles of microRNA-target recognition. PLOS Biol 2005, 3:e85.

24. Grimson A, Srivastava M, Fahey B, Woodcroft BJ, Chiang HR, King N, Degnan BM, Rokhsar DS, Bartel DP: Early origins and evolution of microRNAs and Piwi-interacting RNAs in animals. Nature 2008, 455:1193-U1115.

25. Mastrokolias A, Den Dunnen JT, van Ommen GB, t Hoen PA, Van RoonMom WM: Increased sensitivity of next generation sequencing-based expression profiling after globin reduction in human blood RNA. BMC Genomics 2012, 13:28.

26. Cortez MA, Bueso-Ramos C, Ferdin J, Lopez-Berestein G, Sood AK, Calin GA MicroRNAs in body fluids-the mix of hormones and biomarkers. Nat Rev Clin Oncol 2011, 8:467-477.

27. Kumar S, Hedges SB: TimeTree2: species divergence times on the iPhone. Bioinformatics 2011, 27:2023-2024

28. Friedlander MR, Chen W, Adamidi C, Maaskola J, Einspanier R, Knespel S, Rajewsky N: Discovering microRNAs from deep sequencing data using miRDeep. Nat Biotech 2008, 26:407-415.

29. Gardner PP, Daub J, Tate JG, Nawrocki EP, Kolbe DL, Lindgreen S, Wilkinson AC, Finn RD, Griffiths-Jones S, Eddy SR, Bateman A: Rfam: updates to the RNA families database. Nucleic Acids Res 2009, 37:D136-D140.

30. Griffiths-Jones S, Bateman A, Marshall M, Khanna A, Eddy SR: Rfam: an RNA family database. Nucleic Acids Res 2003, 31:439-441.

31. Griffiths-Jones S, Grocock RJ, van Dongen S, Bateman A, Enright AJ: miRBase: microRNA sequences, targets and gene nomenclature. Nucleic Acids Res 2006, 34:D140-144.

32. Griffiths-Jones S, Saini HK, van Dongen S, Enright AJ: miRBase: tools for microRNA genomics. Nucleic Acids Res 2008, 36:D154-158.

33. Kozomara A, Griffiths-Jones S: miRBase: integrating microRNA annotation and deep-sequencing data. Nucleic Acids Res 2011, 39:D152-157.

34. Griffiths-Jones S: The microRNA registry. Nucleic Acids Res 2004, 32:D109-D111. 
35. Flicek P, Amode MR, Barrell D, Beal K, Brent S, Carvalho-Silva D, Clapham P, Coates G, Fairley S, Fitzgerald S, et al: Ensembl 2012. Nucleic Acids Res 2012, 40:D84-90.

36. Langmead B, Trapnell C, Pop M, Salzberg SL: Ultrafast and memoryefficient alignment of short DNA sequences to the human genome. Genome Biol 2009, 10:R25.

37. Altschul SF, Gish W, Miller W, Myers EW, Lipman DJ: Basic local alignment search tool. J Mol Biol 1990, 215:403-410.

38. Hofacker IL, Stadler PF: Memory efficient folding algorithms for circular RNA secondary structures. Bioinformatics 2006, 22:1172-1176.

39. Hofacker IL: Vienna RNA secondary structure server. Nucleic Acids Res 2003, 31:3429-3431.

40. Friedman RC, Farh $\mathrm{KKH}$, Burge $\mathrm{CB}$, Bartel DP: Most mammalian mRNAs are conserved targets of microRNAs. Genome Res 2009, 19:92-105.

41. Grimson A, Farh KKH, Johnston WK, Garrett-Engele P, Lim LP, Bartel DP. MicroRNA targeting specificity in mammals: determinants beyond seed pairing. Mol Cell 2007, 27:91-105.

42. Lewis BP, Burge CB, Bartel DP: Conserved seed pairing, often flanked by adenosines, indicates that thousands of human genes are microRNA targets. Cell 2005, 120:15-20.

43. Zheng GX, Ravi A, Gould GM, Burge CB, Sharp PA: Genome-wide impact of a recently expanded microRNA cluster in mouse. Proc Natl Acad Sci USA 2011, 108:15804-15809.

44. Trapnell C, Pachter L, Salzberg SL: TopHat: discovering splice junctions with RNA-Seq. Bioinformatics 2009, 25:1105-1111.

45. Kasprzyk A: BioMart: driving a paradigm change in biological data management. Database 2011, 2011:bar049.

46. Kanehisa M, Goto S, Sato Y, Furumichi M, Tanabe M: KEGG for integration and interpretation of large-scale molecular data sets. Nucleic Acids Res 2012, 40:D109-114.

47. Falcon S, Gentleman R: Using GOstats to test gene lists for GO term association. Bioinformatics 2007, 23:257-258.

48. R Core team: $R$ : A language and environment for statistical computing Vienna, Austria: R Foundation for Statistical Computing; 2012.

49. Carlson M: KEGG.db: A set of annotation maps for KEGG; 2013. R package version 2.9.1.

50. Carlson M: org.Bt.eg.db: Genome wide annotation for Bovine; 2013. R package version 2.9.0.

\section{Submit your next manuscript to BioMed Central and take full advantage of:}

- Convenient online submission

- Thorough peer review

- No space constraints or color figure charges

- Immediate publication on acceptance

- Inclusion in PubMed, CAS, Scopus and Google Scholar

- Research which is freely available for redistribution 